\begin{tabular}{ll} 
Journal of Environmental \\
Analysis and Progress \\
ISSN: $2525-815 \mathrm{X}$ & Journal homepage: $w$ ww.jeap.ufrpe.br/ \\
\hline
\end{tabular}

\title{
Estrutura da comunidade de macroinvertebrados bentônicos em nascentes de fragmentos de Mata Atlântica
}

\section{Benthic macroinvertebrates community structure in Atlantic rainforest water sources}

Gabriela Pupo Hagemeyer ${ }^{a}$, Rhuann Carlo Viero Taques ${ }^{a}$, Júlia Laís Yokota Campos ${ }^{\mathrm{a}}$, Gisele Campos $^{\mathrm{a}}$, Ana Lúcia Suriani Affonso ${ }^{\mathrm{a}}$

${ }^{a}$ Universidade Estadual do Centro-Oeste/Unicentro, Departamento de Ciências Biológicas. Rua Alameda Élio Antonio Dalla Vecchia, n. 838, Vila Carli, Guarapuava, Paraná. CEP: 85040-167. E-mail: gabrielahagemeyer@gmail.com, rhuanntaques@gmail.com, juliayokotacampos@gmail.com, giselecampos814@gmail.com, analuciabio@ gmail.com.

A R T I C L E I N F O

Recebido 23 Nov 2020

Aceito 05 Jan 2022

Publicado 29 Jan 2022

\begin{abstract}
A B S T R A C T
The original area of the Mixed Ombrophilous Forest (MAF), Atlantic Rainforest (AR) phytophisiognomy, has been suffering reduction to a small forest fragment, which is caused by anthropic activities. The water sources from these fragments develop a crucial paper to the maintenance of ecosystemic services performed by these biomes, providing the necessary hydric resources to the cities' supply. There are diverse organisms in aquatic ecosystems, like the benthic macroinvertebrates. The goal of this investigation was to compare the fauna structure of these organisms from two water sources, one superficial and another underground, both located in MAF fragments. The sediments were collected using the Surber sampler and the organisms were identified to the smallest taxonomic rank possible. Subsequently, ecological indices calculations were made followed by a community web analysis. Results revealed that the underground water source presented more abundance with 233 collected organisms, where Elmidae (51\%), Chironomidae $(29 \%)$, and Calamoceratidae (10\%) were the most frequent taxa. However, the superficial water source showed a higher diversity value, in which Chironomidae (39\%), Elmidae (15\%), and Polycentropodidae $(10 \%)$ were the most frequent taxons. The taxons Oligochaeta, Elmidae, Parametriocnemus, Stenochironomus, Endotribelus, Caladomyia, and Tipulidae appeared in both water sources. It is concluded that the physical location of the water sources (superficial or underground) influences the diversity of organisms from the same area, even if they belong to preserved forest fragments from the same biome. It shows that habitat heterogeneity and the distinct environmental conditions interfere in the benthic community structure from the water sources.
\end{abstract}

Keywords: Water, Mixed Ombrophilous Forest, underground, superficial, zoobenthos.

\section{R E S U M O}

A Floresta Ombrófila Mista (FOM), fitofisionomia da Mata Atlântica (MA), vem sofrendo redução de sua área original a pequenos fragmentos florestais, o que promove impactos na biodiversidade e no ambiente. As nascentes de água existentes nesses fragmentos desempenham papel crucial para a manutenção dos serviços ecossistêmicos prestados por esse bioma, que fornecem os recursos hídricos necessários ao abastecimento de cidades. Dentre os organismos presentes nesses ecossistemas aquáticos destacam-se os macroinvertebrados bentônicos. O objetivo deste estudo foi comparar a estrutura da fauna destes organismos em duas nascentes, uma superficial e uma subterrânea, ambas localizadas em fragmentos de FOM. O sedimento foi coletado por meio do amostrador Surber e os organismos foram identificados até o menor nível taxonômico possível. Posteriormente, foram realizados cálculos dos índices ecológicos e uma análise de rede para as 
comunidades. Os resultados demonstraram que a nascente subterrânea apresentou maior abundância com 232 organismos coletados, onde Elmidae (51\%), Chironomidae $(29 \%)$ e Calamoceratidae $(10 \%)$ foram os táxons mais frequentes. Porém, a nascente superficial obteve maior valor de diversidade, e os táxons mais frequentes foram Chironomidae (39\%), Elmidae (15\%) e Polycentropodidae (10\%). Os táxons Oligochaeta, Elmidae, Parametriocnemus, Stenochironomus, Endotribelus, Caladomyia e Tipulidae foram compartilhados por ambas as nascentes. Conclui-se que mesmo pertencendo a fragmentos florestais preservados e de um mesmo bioma, a localização física da nascente (superficial ou subterrânea) faz com que os organismos habitantes sejam diversos. Isso demonstra que a heterogeneidade de habitats e as condições ambientais distintas interferem na estrutura da comunidade bentônica existente em nascentes.

Palavras-Chave: Água, Floresta Ombrófila Mista, subterrânea, superficial, zoobentos.

\section{Introdução}

A Mata Atlântica (MA) possui elevada riqueza de espécies (animais e vegetais) sendo considerada um dos 25 hotspots de biodiversidade no mundo (Tabarelli et al., 2005). Porém, de acordo com estudos recentes, este bioma brasileiro encontra-se ameaçado, devido principalmente as atividades antrópicas associadas a produção em massa e que visam somente ao lucro (Fundação SOS Mata Atlântica, 2020). Segundo a Atlas dos Remanescentes Florestais da Mata Atlântica, nos últimos dez anos houve um aumento de cerca de $27,2 \%$ na taxa de desmatamento desta mata e, consequentemente em fragmentação florestal, assegurando alterações expressivas nas (inter)relações das espécies para com seus habitats (Fundação SOS Mata Atlântica, 2020).

$\mathrm{Na}$ composição fitofisionômica da MA evidenciam-se diferentes formações florestais nativas como a Floresta Ombrófila Densa, Floresta Ombrófila Mista (FOM), Floresta Ombrófila Aberta, Floresta Estacional Semidecidual e Floresta Estacional Decidual, além de ecossistemas associados como manguezais, restingas, campos de altitude, brejos interioranos e encraves florestais (Sonego, Backes \& Souza, 2007). Dentro deste contexto, a FOM sobreleva-se por apresentar um regime pluviométrico relativamente alto aproximadamente $2.252 \mathrm{~mm} \cdot \mathrm{ano}^{-1} \quad$ (Sonego, Backes \& Souza, 2007) - com a presença matizada de espécies de angiospermas e gimnospermas, com destaque ao Pinheiro do Paraná (Araucaria angustifolia (Bertol.) Kuntze, 1898) (Fundação SOS Mata Atlântica, 2020).

Os fragmentos florestais de MA, remanescentes no estado do Paraná, são comumente preservados por meio de Unidades de Conservação (UCs). Nestes ambientes, as nascentes de água possuem papéis centrais no abastecimento das bacias hidrográficas locais. De acordo com o Código Florestal Brasileiro, uma nascente se caracteriza por um "afloramento natural do lençol freático que apresenta perenidade e dá início a um curso d'água" (Brasil, 2012, Art. $3^{\circ}$, item XVII). Diante disto, o estudo da qualidade da água para o manejo e proteção destes fragmentos florestais é essencial para que a manutenção da biodiversidade local e dos serviços ecossistêmicos prestados pelos ecossistemas aquáticos. Dentre estes destacamos os serviços de provisão (qualidade e quantidade da água potável), regulação (autodepuração de poluentes), culturais (recreação, apreciação estética) e de suporte (ciclagem de nutrientes, produção de oxigênio, entre outros).

De acordo com o Sistema Nacional de Unidades de Conservação (SNUC), uma UC é definida por "espaço territorial e seus recursos ambientais, incluindo as águas jurisdicionais, com características naturais relevantes, legalmente instituído pelo Poder Público, com objetivos de conservação e limites definidos, sob regime especial de administração, ao qual se aplicam garantias adequadas de proteção" (Brasil, 2000, Art. $2^{\circ}$, item I). Além disso, elas são divididas em 12 categorias, sendo que cada uma possui características específicas acerca de seus objetivos para a preservação e uso do local, ou seja, existem aquelas que exibem maior restrição quanto a visitação e uso de recursos naturais, e aquelas em que ocorre a conservação juntamente com o uso sustentável de recursos naturais (Brasil, 2000).

De acordo com Vallejo (2003) uma UC está ligada à preservação das necessidades básicas de sobrevivência das múltiplas espécies nos ecossistemas. Portanto, não se deve dar somente importância ao espaço físico ocupado, mas sim analisar o ambiente como um conjunto de fatores que se relacionam entre si, levando em conta a função dos organismos no ecossistema (Odum, 1977).

As UCs em estudo são categorizadas pelo Artigo $8^{\circ}$ do SNUC (Lei 9.985/2000) como Estação Ecológica, que "tem como objetivo a preservação da natureza e a realização de pesquisas científicas" (Brasil, 2000, Art. $9^{\circ}$ ). O 
artigo $9^{\circ}$ dessa lei define como características e atribuições de uma Estação Ecológica: posse e domínio público, onde é proibida a visitação pública (exceto com objetivo educacional, seguindo um plano de manejo), a pesquisa científica necessita de autorização prévia para fazer qualquer ação no local, e só é permitido que ocorram alterações dos ecossistemas se forem obedecidas medidas que visem restaurar $o$ ecossistemas modificados, preservar a diversidade biológica, e a coleta de componentes com finalidades científicas.

Dentre os organismos que habitam os ecossistemas aquáticos, destacam-se os macroinvertebrados bentônicos. Estes organismos habitam o sedimento de corpos d’água e apresentam táxons sensíveis ou tolerantes à poluição e à degradação ambiental. Desta forma, são utilizados como bioindicadores e nos fornecem informações sobre o grau de preservação ou de impacto destes locais. A utilização destes organismos pela comunidade científica deve-se a diversos fatores como o ciclo de vida suficientemente longo, fácil amostragem, técnicas e metodologias já padronizadas, custo relativamente baixo e alta diversidade taxonômica (Cummins, 1996; Callisto, Moretti \& Goulart, 2001). Além disso, essa comunidade possui uma elevada representatividade de táxons que apresentam tolerância ou não a diferentes níveis de contaminação (Gonçalves \& Aranha, 2004).

Apesar de serem muito utilizados em estudos de biomonitoramento, na avaliação da saúde, manejo e gestão de ambientes, pesquisas sobre estes organismos em nascentes são escassas, quando comparamos a córregos ou lagoas (Piedras et al., 2006; Copati, Schirmer \& Machado 2010; Pego, Santos \& Santos, 2010; Santos \& Melo, 2017; Azevedo \& Melo, 2018).

Diante do exposto, o presente estudo buscou realizar um levantamento de macroinvertebrados bentônicos em duas nascentes presentes em dois fragmentos florestais de FOM, localizados no interior do Paraná. A hipótese inicial da pesquisa é de que ambas as nascentes apresentam diversidade de táxons e de grupos tróficos funcionais, com diferentes dinâmicas organismos/ambiente, já que uma delas encontrase na porção superficial do solo e a outra é subterrânea.

Esta pesquisa justifica-se por subsidiar informações sobre a biodiversidade aquática de nascentes, fato este que poderá contribuir para a gestão e o manejo de UCs. Além disso, ela apresenta a comunidade bentônica como uma nova métrica biológica que poderá ser utilizada como critério nas tábuas de avaliações do ICMS Ecológico por Biodiversidade. Este último, caracteriza-se como um instrumento de política pública, que fornece recursos financeiros aos municípios que abrigam e preservam em seu território UCs e mananciais (IAT, 2021).

\section{Material e Métodos \\ Área de estudo \\ $\mathrm{O}$ estudo foi realizado em duas nascentes, uma localizada na porção superficial do solo e outra subterrânea, presente em diferentes fragmentos florestais de MA na região centro-sul do Estado do Paraná, Brasil (Figura 1).}

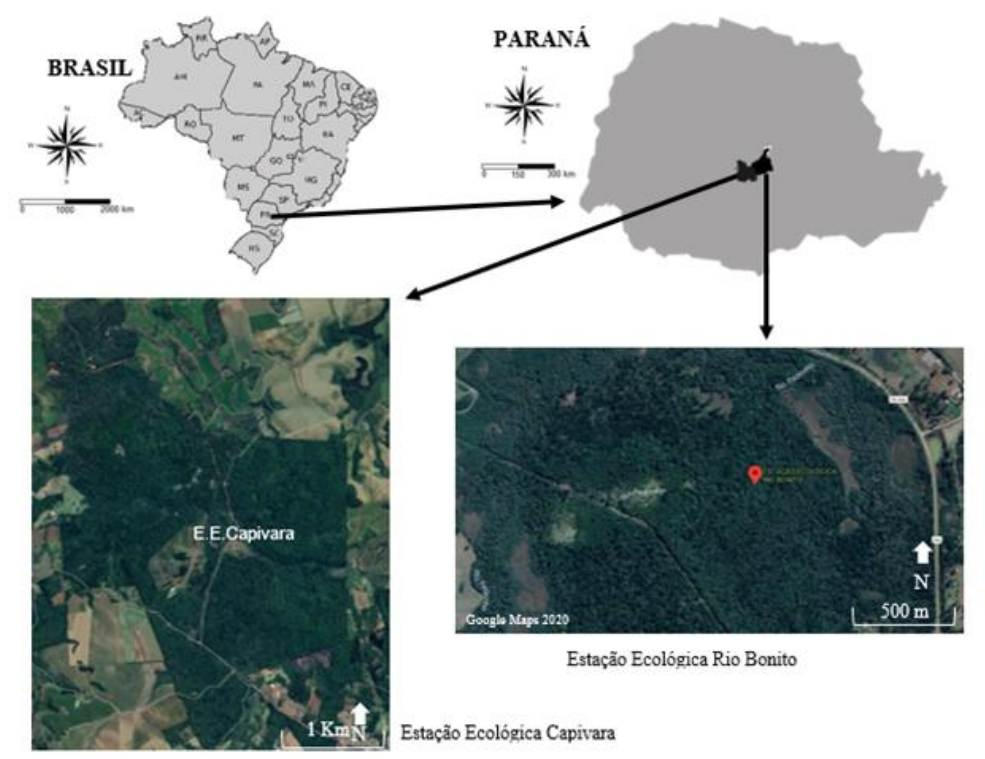

Figura 1. Localização das nascentes pesquisadas na Estação Ecológica Rio Bonito (Turvo, Paraná) e na Estação Ecológica Capivara II (Campina do Simão, Paraná). Fonte: Hagemeyer et al. (2020). 
As amostragens da fauna bentônica e da água desses locais foram realizadas em setembro de 2019. A nascente superficial está inserida na Estação Ecológica (EE) Capivara II entre as coordenadas geográficas $\left(25^{\circ} 04,43.53\right.$ ' $\mathrm{S}$ e $51^{\circ} 49^{\prime} 27.89^{\prime}$ ' W), no município de Campina no Simão, PR. A nascente subterrânea, por sua vez, encontra-se na EE Rio Bonito, município de

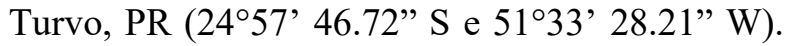
Uma distância de, aproximadamente, 70 quilômetros separa os municípios, porém, ambos os locais se constituem fragmentos florestais de FOM. De acordo com Alvares et al. (2014), a região possui a predominância do clima subtropical mesotérmico, com verão brando e inverno moderado, além de possuir algumas épocas de geada e chuvas periódicas.

\section{Coleta de dados}

Para a coleta do sedimento foi utilizado o Surber (área 0,09 $\mathrm{m}^{2}$ ), onde o material amostrado foi acondicionado em sacos plásticos com formol (7\%), para posterior lavagem. A lavagem do material coletado ocorreu no laboratório de Ecologia de Bentos da Universidade Estadual do Centro Oeste, no Campus Centro de Desenvolvimento Educacional e Tecnológico de Guarapuava (Cedeteg); o processo foi feito com auxílio de duas peneiras, uma com $1 \mathrm{~mm} \mathrm{\mu m}^{-1} \mathrm{e}$ a outra com $250 \mathrm{~mm} \mathrm{\mu m}^{-1}$ de abertura de malha. $\mathrm{O}$ material foi acondicionado em um recipiente com álcool $(70 \%)$ para posterior triagem. Para a triagem foi utilizada uma bandeja transiluminada e os organismos presentes foram inseridos em recipientes de vidro com álcool $(70 \%)$ para serem identificados.

A identificação dos macroinvertebrados ocorreu por meio de um microscópio estereoscópio e um microscópio óptico até o menor nível taxonômico possível, a partir das chaves de identificação de Epler (2001), Mugnai, Nessimian \& Baptista (2010) e Trivinho-Strixino (2011). Os organismos da família Chironomidae são considerados os mais abundantes nas comunidades bentônicas (Callisto et al., 2002), sendo encontrados em sedimentos com matéria orgânica em decomposição (Amorim \& Castillo, 2009) e, dessa forma, podem estar presentes, tanto em ambientes poluídos, como ambientes preservados. Dessa forma, os mesmos foram identificados até gênero, ampliando a caracterização da fauna local, além de ampliar a caracterização da comunidade no seu todo.

\section{Análises dos dados}

A estrutura das comunidades de macroinvertebrados bentônicos das nascentes foi avaliada por meio dos cálculos dos índices de diversidade de Shannon-Wiener (H') (Krebs, 1999), equidade de Pielou (J') e dominância taxonômica, que foram calculados utilizando o software Dives 2.0. Para demostrar a existência ou não de táxons comuns foi realizada a análise de rede, por meio do software Gephi.

As categorias tróficas dos macroinvertebrados bentônicos identificados seguiram aquelas descritas por Merritt \& Cummins (1996) sendo: I) fragmentadores (ou retalhadores), incluindo herbívoros e comedores de grandes partículas orgânicas; II) coletores, incluindo filtradores e roçadores de pequenas partículas; III) raspadores de algas perifíticas e IV) predadores.

\section{Resultados e Discussão}

Foram registrados em ambas as nascentes 415 organismos bentônicos pertencentes a 32 táxons dos filos Anellida e Arthropoda, onde este último foi grupo mais representativo (Tabela 1). As ordens mais abundantes foram Coleoptera (Turvo $\mathrm{n}=190$ indivíduos; Campina do Simão $\mathrm{n}$ $=38$ indivíduos), Diptera (Turvo $\mathrm{n}=6$ indivíduos; Campina do Simão $\mathrm{n}=91$ indivíduos) $\mathrm{e}$ Trichoptera (Turvo $\mathrm{n}=23$ indivíduos; Campina do Simão $n=22$ indivíduos).

Tabela 1. Organismos bentônicos registrado em nascentes (subterrânea e superficial) de fragmentos de FOM nos Municípios de Turvo e Campina do Simão, Paraná, e suas classificações tróficas. Fonte: Hagemeyer et al. (2020).

\begin{tabular}{lccc}
\hline Taxa & $\begin{array}{c}\text { Nascente Subterrânea } \\
\text { (Turvo) }\end{array}$ & $\begin{array}{c}\text { Nascente Superficial } \\
\text { (Campina do Simão) }\end{array}$ & $\begin{array}{c}\text { Classificação } \\
\text { Trófica }\end{array}$ \\
\hline Filo Annelida & 12 & 8 & Coletor-catador \\
$\begin{array}{l}\text { Classe Oligochaeta } \\
\text { Filo Arthropoda }\end{array}$ & & & \\
Ordem Coleoptera & 0 & 1 & Predador \\
Família Dytiscidae & 118 & 27 & Raspador \\
Família Elmidae & 0 & 2 & Predador \\
Família Lutrochidae & 0 & 1 & Predador \\
Família Noteridae & & &
\end{tabular}


Família Psephenidae

Família Dryopidae

Ordem Crustacea

Família Hyalellidae

Ordem Diptera

Família Ceratopogonidae

Família Chironomidae Caladomyia Säwedall

(1981)

Cardiocladius Kieffer

(1912)

Chironomus Meigen

(1803)

Cricotopus Wulp (1874)

Djalmabatista Fittkau

(1968)

Endotribelus Grodhaus

(1987)

Metriocnemus Meigen

(1818)

Parametriocnemus Goetghel

(1932)

Paraphaenocladius

Thienemann (1924)

Paratanytarsus Bause

(1913)

Paratendipes Kieffer

(1911)

Pentaneura Philippi (1865)

Polypedilum Kieffer

(1912)

Stenochironomus Kieffer

(1919)

Tanytarsus Wulp (1874)

Família Tabanidae

Família Tipulidae

Ordem Decapoda

Família Aeglidae

Ordem Hemiptera

Família Mesoveliidae

Família Veliidae

Ordem Lepidoptera

Família Piralydae

Ordem Odonata

Família Libellulidae

Ordem Trichoptera

Família Hidropsychidae

Família Philopotamidae

Família Polycentropodidae

Família Calamoceratidae

Ordem Amphipoda

Família Hyalellidae

TOTAL

232

0

Raspador

Predador

Coletor-catador

Predador

Coletor-catador

Coletor-catador

Coletor-catador

Coletor-catador

Coletor-catador

Coletor-catador

Coletor-catador

Coletor-catador

Coletor-catador

Coletor-catador

Coletor-catador

Coletor-catador

Coletor-catador

Coletor-catador

Coletor-catador

Coletor-catador

Triturador

Triturador

Coletor-catador

Predador

Predador

Fragmentador

Predador

Predador

Coletor-catador

Predador

Coletor-catador

Triturador
A nascente subterrânea (Turvo, PR) apresentou maior abundância com 232 organismos coletados, distribuídos em 12 táxons
(Tabela 1). Nessa nascente os táxons mais frequentes foram Elmidae (51\%), Chironomidae (29\%) e Calamoceratidae (10\%) (Figura 2). 


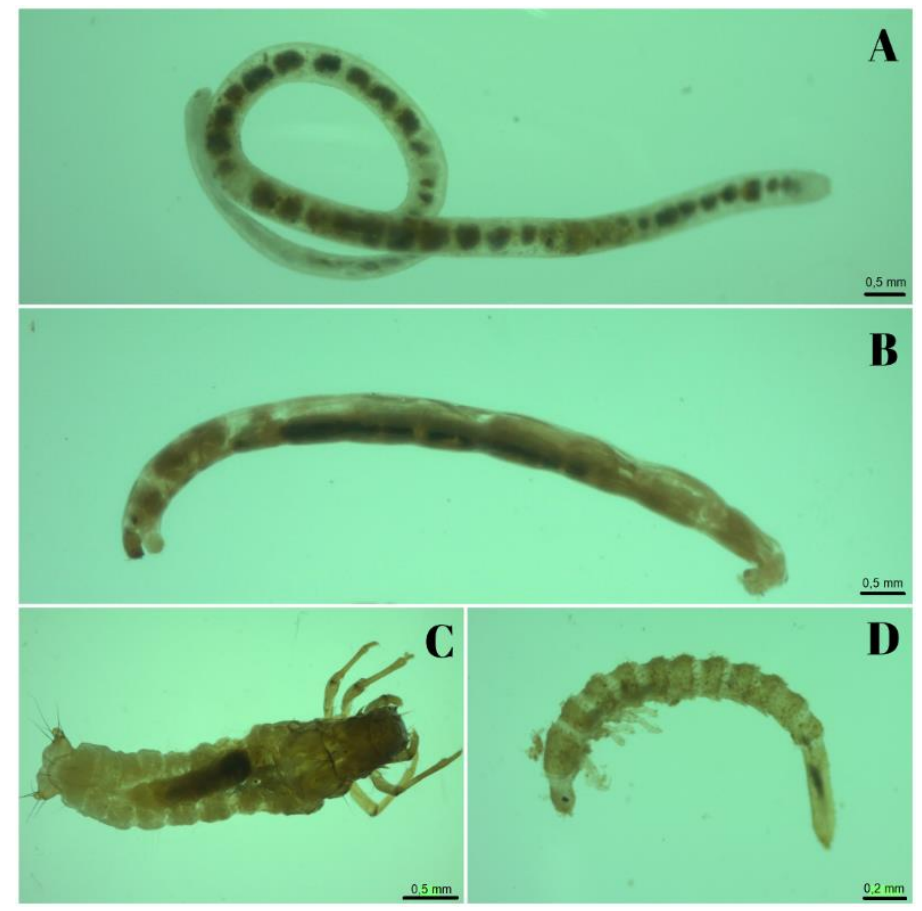

Figura 2. Fotografias de representantes dos táxons (A) Oligochaeta; (B) Chironomidae registrados na nascente superficial no município de Campina do Simão/PR e (C) Calamoceratidae; (D) Elmidae, registrados na nascente subterrânea no município de Turvo/PR. Fonte: Hagemeyer et al. (2020).

$\mathrm{Na}$ nascente localizada na superfície do solo (Campina do Simão, PR) os táxons mais frequentes foram Chironomidae (39\%), Elmidae $(15 \%)$ e Polycentropodidae (10\%) (Figura 2).

A família Polycentropodidae é considerada uma ótima indicadora de ambientes com pouca eutrofização, vivendo em águas limpas e bem oxigenadas (Callisto, Moretti \& Goulart, 2001). Os indivíduos da família Chironomidae possuem adaptações fisiológicas, morfológicas e comportamentais que tornam sua sobrevivência melhor em diversas condições ambientais (Merrit \& Cummins, 1996). A família Elmidae, apesar de apresentar resistência a áreas impactadas, é registrada na literatura com a preferência por áreas mais íntegras (Goulart \& Callisto, 2003; Piedras et al., 2006). As larvas da família Calamoceratidae usam as folhas caídas para construção de abrigos e como fonte de alimento e são consideradas organismos sensíveis à poluição (Metcalf, 1989; Resh \& Jackson, 1993). Organismos coletores-catadores, como os Oligochaeta e Chironomidae, ocorreram com maior abundância absoluta na nascente subterrânea (Tabela 1). Estes macroinvertebrados alimentam-se de pequenas partículas de matéria orgânica nos depósitos de sedimento (Merritt \& Cummins, 1996). De acordo com estudos, o constante aporte de matéria orgânica de origem alóctone como folhas, frutos e galhos provenientes da densa vegetação do entorno podem favorecer o estabelecimento de organismos com esta típica trofia (Marques, Barbosa \&
Callisto, 1999; Silva et al., 2009; Barbola et al., 2011). Deste modo, a matéria orgânica se torna um alimento passível de repartição entre inúmeros organismos, onde pode ser considerada como um recurso ilimitado nesses ecossistemas (Motta \& Uieda, 2004). Além disso, apesar de registrada a existência de organismos predadores e trituradores, a presença destes é limitada, já que com a elevada presença de Chironomidae pode ocorrer uma competição por recursos alimentares, como o perifíton por exemplo, além de competir também por abrigo e proteção (Amorin \& Castillo, 2009).

$\mathrm{Na}$ nascente localizada superficialmente ao solo, os organismos predadores como Hemiptera, Odonata e Trichoptera foram mais abundantes (Tabela 1). Silva et al. (2009) destacam que os macroinvertebrados bentônicos destes últimos dois táxons são tradicionalmente conhecidos como predadores, especialmente de larvas de Chironomidae, justificando a menor abundância de organismos coletores-catadores neste ponto amostral. Desta forma, esses resultados não refletem diretamente a influência de matéria orgânica de origem alóctone, como sugerido na pesquisa de Chagas et al. (2017).

Os macroinvertebrados, pertencentes a guilda dos fragmentadores, foram registrados apenas na nascente superficial. Estes possuem papel de reduzir a matéria orgânica em partículas menores (Silva et al., 2009), porém, somente o fazem quando os recursos alimentares mostraram alguma mudança estrutural ou bioquímica, de 
modo a tornar-se palatável (Cummins, 1996). Moretti (2005). Porém, este processo pode demorar semanas, ou até mesmo meses, e, portanto, a ausência desta guilda na nascente subterrânea pode ocorrer pela ausência de matéria orgânica palatável de origem alóctone.

Ambas as nascentes, a subterrânea e a superficial, encontram-se em fragmentos de MA, entretanto, suas composições taxonômicas de macroinvertebrados bentônicos apresentaram-se como diferentes estatisticamente $(p<0,05)$, demonstrando que as faunas bentônicas diferem pelo ambiente físico ocupado por cada uma delas.
É importante destacar que essas diferenças podem decorrer de fatores como luminosidade do local (nascente subterrânea), oxigenação dessas águas, pois em ambientes com menor subterrâneos, a comunidade fitoplanctônica é afetada, inferindo diretamente na concentração de oxigênio dissolvido no local e na alimentação de alguns macroinvertebrados bentônicos. O valor máximo para diversidade e equitabilidade foram observados na nascente superficial, enquanto a nascente subterrânea apresentou maior dominância taxonômica (Tabela 2).

Tabela 2. Índice de diversidade de Shannon (H'), Equitabilidade de Pielou (J) e Dominância taxonômica de macroinvertebrados bentônicos presentes em duas nascentes (subterrânea a superficial) de fragmentos de MA. Fonte: Hagemeyer et al. (2020).

\begin{tabular}{ccc}
\hline & Nascente subterrânea & Nascente superficial \\
\hline Diversidade $\left(\mathrm{H}^{\prime}\right)$ & 1.43 & 2.18 \\
Equitabilidade $(\mathrm{J})$ & 0.57 & 0.73 \\
Dominância & 0.33 & 0.18 \\
\hline
\end{tabular}

Sete táxons foram compartilhados por ambas as nascentes sendo eles: Oligochaeta, Elmidae, Parametriocnemus, Stenochironomus, Endotribelus, Caladomyia e Tipulidae (Figura 3). Os Oligochaeta aquáticos são bastante sensíveis a perturbações antrópicas, e, portanto, podem indicar o status-quo do ambiente (Takeda et al., 2017). Estudos sugerem que quando presentes apontam locais de vegetação primária e bom estado de conservação (Edwards \& Bohlen, 1996; Piedras et al., 2006; Pimenta et al., 2016). Chagas et al. (2017) destacam que organismos raspadores como Elmidae podem fornecer informações sobre a qualidade ambiental de ecossistemas lóticos. Além disso, a ordem Coleoptera, em geral, pode ser encontrada em praticamente todos os meios (Silva \& Silva, 2011) já que seus organismos participam ativamente nos processos de ciclagem de nutrientes nos ambientes onde habitam (Davis et al., 2001). O registo de Tipulidae em ambas as nascentes, por sua vez, demonstra que estes são encontrados, em sua grande maioria, em áreas ribanceiras (Silveira, Buss \& Baptista 2006), uma vez que também dependem de matéria orgânica alóctone.

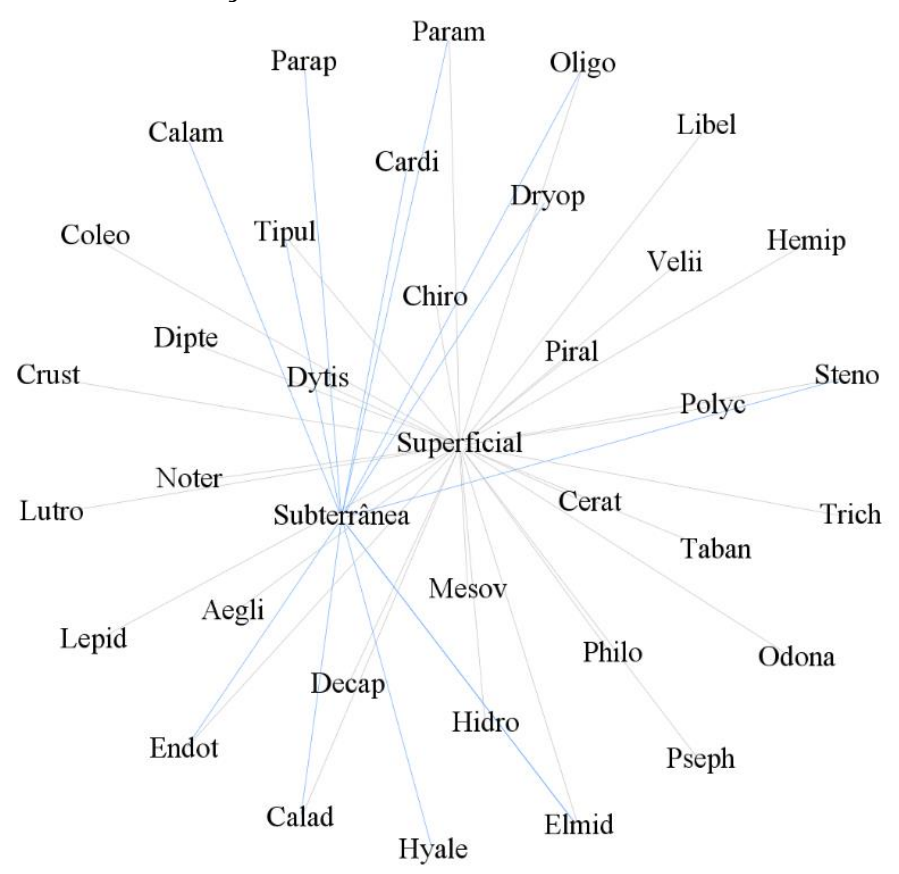

Figura 3. Análise de rede evidenciando-se os táxons exclusivos e compartilhados pelas nascentes dos municípios de Turvo (subterrânea) e de Campina do Simão (superficial), Paraná. Aegli - Aeglidae; Calad - 
Caladomyia; Calam - Calamoceratidae; Cardi - Cardiocladius; Cerat - Ceratopogonidae; Chiro Chironomus; Coleo - Coleoptera; Crico - Cricotopus; Crusta - Crustacea; Djalm - Djalmabatista; Dryop Dryopidae; Dysti - Dytiscidae; Elmid - Elmidae; Endo - Endotribelus; Hyale - Hyalellidae; Hydro Hydropsychidae; Libel - Libellulidae; Lutro - Lutrochidae; Mesov - Mesoveliidae; Metrio - Metriocnemus; Noter - Noteridae; Oligo - Oligochaeta; Param - Parametriocnemus; ; Parap - Paraphaenocladius; Parat Paratanytarsus; Parap - Paratendipes; Penta - Pentaneura; Philo - Philopotamidae; Polyc Polycentropodidae; Poly - Polypedilum; Pseph - Psephenidae; Piral - Piralydae; Steno - Stenochironomus; Taban - Tabanidae; Tanyt - Tanytarsus; Tipul - Tipulidae; Velii - Veliidae. Fonte: Hagemeyer et al. (2020).

Podemos inferir que é de extrema importância a conservação das nascentes, uma vez que elas são instrumentos essenciais para a manutenção e para a qualidade de vida da população. Além disso, elas realizam a formação de novos cursos d'água por meio do abastecimento dos córregos e riachos, que por sua vez culminam em rios principais. Dessa forma, estudos como este de nascentes, nos possibilita conhecer e utilizar a biota local para realizar pesquisas com indicadores biológicos, os quais, juntamente com os parâmetros físico-químicos, são capazes de refletir a saúde ambiental dos ecossistemas aquáticos (Metcalfe, 1989; Amorim \& Castillo, 2009; Copatti, Schirmer \& Machado, 2010; Pego, Santos \& Santos, 2010; Pimenta et al., 2016; Chagas et al., 2017; Santos \& Melo, 2017). Assim, a qualidade da água, somada à biodiversidade da fauna existente, demonstra a integridade biótica do local (Callisto, Moretti \& Goulart, 2001).

\section{Conclusão}

Apesar de ambas as nascentes pertencerem a fragmentos florestais de FOM, as suas composições faunísticas de macroinvertebrados bentônicos foram distintas (nascente superficial com 32 táxons e nascente subterrânea com 12 táxons). A localização física de cada nascente, uma sobre o solo e a outra no subsolo, pode ter contribuído para este resultado, evidenciando-se também, a resiliência da comunidade bentônica em diferentes habitats, em especial, aqueles que se encontram no interior de UCs.

Por meio desta pesquisa pode-se perceber que o registro qualiquantitativo, bem como investigações de parâmetros ecológicos de macroinvertebrados bentônicos em nascentes são fundamentais para o monitoramento e manejo ambiental de UCs. Apesar das tábuas de Avaliação das Áreas Protegidas cadastradas no ICMS Ecológico levarem em conta a presença de táxons raros, ameaçados ou sob ameaça nas UCs, recomenda-se o enquadramento da composição taxonômica de macroinvertebrados bentônicos, como parâmetro de avaliação, visando um incremento dos índices deste instrumento de política ambiental.

\section{Agradecimentos}

À Universidade Estadual do CentroOeste-Unicentro pela infraestrutura fornecida e às prefeituras municipais de Campia do Simão e de Turvo-PR pelo financiamento parcial da pesquisa, advindo dos fundos do ICMS Ecológico por Biodiversidade (Convênios no 99/2017 e no 124/ 2018).

\section{Referências}

Alvares, C. A.; Stape, J. L.; Sentelhas, P. C.; Gonçalves, J. L. M.; Sparovck, G. 2014. Koppen's climate classification map for Brazil. Meteorologische Zeitschrift, 22, 6, 711-728.

Amorim, A. C. F.; Castillo, A. R. 2009. Macroinvertebrados bentônicos como bioindicadores da Qualidade da água do baixo rio Perequê, Cubatão, São Paulo, Brasil. Biodiversidade Pampeana, 7, 1, 16-22.

Azevedo, V. S.; Melo, T. G. G. 2018. Macroinvertebrados bentônicos como ferramenta na avaliação da qualidade ambiental de corpos d'água do Parque Shangrilá. XXIX Congresso Nacional de Saneamento e Meio Ambiente. AESABESP, Associação dos Engenheiros da Sabesp. Available at: https://www.tratamentodeagua.com.br/artigo /macroinvertebrados-qualidade-corposdagua/. Access at: August 02, 2020.

Barbola, I. F.; Moraes, M. F. P. G.; Anazawa, T. M.; Nascimento, E. A.; Sepka, E. R.; Polegatto, C. M. 2011. Avaliação da comunidade de macroinvertebrados aquáticos como ferramenta para o monitoramento de um reservatório na bacia do Rio Pitangui, Paraná, Brasil. Iheringia, 101, 2, 15-23.

Brasil. Decreto $\mathrm{n}^{\circ}$ 12.651, 25 de maio de 2012. Decreto do Código Florestal. Available at: http://www.planalto.gov.br/ccivil_03/_Ato20 11-2014/2012/Lei/L12651.htm. Access at: July 07, 2020. 
Brasil. Lei Federal $n^{\circ}$ 9.985, 2000. Institui o Sistema Nacional de Unidades de Conservação da Natureza-SNUC, estabelece critérios e normas para a criação, implantação e gestão das unidades de conservação. Available at: http://www.planalto.gov.br/ccivil_03/leis/L9 985.htm. Accessed in: July 07, 2020.

Callisto, M.; Ferreira, W.; Moreno, P.; Goulart, M. D. C.; Petrucio, M. 2002. Aplicação de um protocolo de avaliação rápida da diversidade de habitats em atividades de ensino e pesquisa (MG-RJ). Acta Limnologica Brasiliensis, 14, 1, 91-98.

Callisto, M.; Moretti, M.; Goulart, M. 2001. Macroinvertebrados bentônicos como ferramenta para avaliar a saúde de riachos. Revista Brasileira de Recursos Hídricos, 6, 1, 71-82.

Chagas, F. B.; Rutkoski, C. F.; Bieniek, G. B.; Vargas, G. D. L. P.; Hartmann, P. A.; Hartmann, M. T. 2017. Utilização da estrutura de comunidades de macroinvertebrados bentônicos como indicador de qualidade da água em rios no sul do Brasil. Ambiente \& Água, 12, 3, 217 415.

Copatti, C. E.; Schirmer, F. G.; Machado, J. V. V. 2010. Diversidade de macroinvertebrados bentônicos na avaliação da qualidade ambiental de uma microbacia no sul do Brasil. Perspectiva, 34, 125, 79-91.

Cummins, K. W. 1996. An introduction to the aquatic insects of North America. Dubuque: Kendall/Hunt. 1498p.

Davis, A. J.; Holloway, J. D.; Huijbregts, H.; Krikken, J.; Kirk-Spriggs, A. H.; Sutton, S. L. 2001. Dung beetles as indicators of change in the forests of northern Borneo. Journal of Applied Ecology, 38, 593-616.

Edwards, C. A.; Bohlen P. J. 1996. Biology and ecology of earthworms. Chapman and Hall, $3^{\mathrm{a}}$ Edition, London.

Epler, J. H. 2001. Identification manual for the larval Chironomidae (Diptera) of North and South Carolina. North Carolina Department of Environmental and Natural Resources Division of Water Quality, 528p.

Fundação SOS Mata Atlântica. INPE - Instituto Nacional de Pesquisas Espaciais. Atlas dos remanescentes florestais da mata atlântica período 2018-2019. Relatório técnico. Available at: https://www.sosma.org.br/wpcontent/uploads /2020/06/2020_Atlas_Mata_Atlantica_20182019_relatorio_tecnico_final-1.pdf. Access at: January 15, 2020.
Gonçalves, F. B.; Aranha, J. M. R. 2004. Ocupação espaço temporal pelos macroinvertebrados bentônicos na bacia do rio Ribeirão, Paranaguá, PR (Brasil). Acta Biológica Paranaense, 33, 181-191.

Goulart, M.; Callisto, M. 2003. Bioindicadores de qualidade de água como ferramenta em estudos de impacto ambiental. Revista da FAPAM, 2, 1, 153-164.

IAT. Instituto Água e Terra. ICMS Ecológico por Biodiversidade. 2021. Available at: http://www.iat.pr.gov.br/Pagina/ICMSEcologico-por-Biodiversidade. Access at: January 06, 2021.

Krebs, C. J. 1999. Ecological Methodology. Addison Wesley Longman Inc., $2^{\text {a }}$ Edition, pp. 650 .

Marques, M. M. G. S. M.; Barbosa, F. A. R.; Callisto, M. 1999. Distribution and abundance of Chironomidae (Diptera, Insecta) in South-East Brazil. Brazilian Journal of Biology, 59, 4, 1-13.

Merritt, R. W.; Cummins, K. W. 1996. An introduction to the aquatic insects of North America. Third Edition, Dubuque, Kendall/Hunt, 722p, 826p.

Metcalfe, J. L. 1989. Biological water quality assessment of running waters based on macroinvertebrates communities: history and present status in Europe. Environmental Pollution, 60, 101-139.

Moretti, M. S. 2005. Decomposição de detritos foliares e sua colonização por invertebrados aquáticos em dois córregos na Cadeia do Espinhaço (MG). Dissertação de Mestrado, Universidade Federal de Minas Gerais. Belo Horizonte, Minas Gerais. 63p.

Motta, R. L.; Uieda, V. S. 2004. Diet and trophic groups of an aquatic insect community in a tropical stream. Brazilian Journal of Biology, 64, 4, 809-817.

Mugnai, R.; Nessimian, L. J.; Baptista, D. F. 2010. Manual de identificação de macroinvertebrados aquáticos do estado Rio de Janeiro. $1^{\text {a }}$ Ed., Rio de Janeiro.

Odum, E. P. 1977. Ecologia. Guanabara, Third Edition, São Paulo, 142p.

Pego, A. P.; Santos, L. B.; Santos, J. C. 2010. Macroinvertebrados bentônicos como bioindicadores da qualidade da água em três nascentes do Complexo Parque do Sabiá, Uberlândia, Minas Gerais. Journal of Environmental Analysis and Progress, 6, 4, 26-38.

Piedras, R. S. N.; Bager, A.; Moraes, P. R. R.; Isoldi, L. A.; Ferreira, O. G. L.; Heemann, C. 2006. Macroinvertebrados bentônicos como 
indicadores de qualidade de água na Barragem Santa Bárbara, Pelotas, RS, Brasil. Ciência Rural, 36, 2, 494-500.

Pimenta, S. A.; Boaventura, G. R.; Pena, A. P.; Ribeiro, T. G. 2016. Estudo da qualidade da água por meio de bioindicadores bentônicos em córregos da área rural e urbana. Ambiente \& Água, 11, 1, 198-210.

Resh, V. H.; Jackson, J. K. 1993. Rapid assessment approaches to biomonitoring using benthic macroinvertebrates. Freshwater Biomonitoring and Benthic Macroinvertebrates. In: Rosenberg, D. M.; Resh, V. H. (Eds). Chapman and Hall, New York, pp. 195-233.

Santos, M. O; Melo, S. M. 2017. Influência do uso e ocupação do solo na qualidade da água de nascentes - Macroinvertebrados bentônicos como bioindicadores. Journal of Environmental Analysis and Progress, 2, 01, 36-43.

Silva, F. L.; Pauleto, G. M.; Talamoni, J. L. B.; Ruiz, S. S. Categorização funcional trófica das comunidades de macroinvertebrados de dois reservatórios na região Centro-Oeste do Estado de São Paulo, Brasil. Acta Scientiarum, 31, 1, 73-78.

Silva, P. G.; Silva, F. G. C. 2011. Besouros (Insecta: Coleoptera) utilizados como bioindicadores. Revista Congrega URCAMP, 5, 1, 1-16.
Silveira, M. P.; Buss, D. F.; Baptista, D. F. 2006. Spatial and temporal distribution of benthic macroinvertebrates in a southeastern Brazilian river. Brazilian Journal of Biology, 66, 623-632.

Sonego, E. C.; Backes, A.; Souza, A. F. 2007. Descrição da estrutura de uma Floresta Ombrófila Mista, RS, Brasil, utilizando estimadores não-paramétricos de riqueza e rarefação de amostras. Acta botânico brasileiro, 21, 4, 943-955.

Tabarelli, M.; Pinto, L. P.; Silva, J. M. C.; Hirota, M. M.; Bedê L. C. 2005. Desafios e oportunidades para a conservação da biodiversidade na Mata Atlântica brasileira. Megadiversidade, 1, 1, 132-138.

Takeda, A. M; Fujita, D. S.; Ragonha, F. H.; Petsch, D. K.; Martins, M. C. M. 2017. Oligochaeta (Annelida) de ambientes aquáticos continentais do Estado do Mato Grosso do Sul (Brasil). Iheringia, 107, 0105.

Trivinho-Strixino, S. 2011. Larvas de Chironomidae: Guia de Identificação. São Carlos, Depto. Hidrobiologia/Lab. Entomologia Aquática/UFSCar. 2011.

Vallejo, L. R. 2003. Unidades de conservação: Uma discussão teórica à luz dos conceitos de território e de políticas públicas. GEOgraphia (UFF), 4, 8, 77-106. 